\title{
COMMUTATORS OF MARCINKIEWICZ INTEGRALS ASSOCIATED WITH SCHRÖDINGER OPERATOR ON GENERALIZED WEIGHTED MORREY SPACES
}

\section{Vagif S. Guliyev, Ali Akbulut, Vugar H. Hamzayev and Okan Kuzu}

Abstract. Let $L=-\Delta+V$ be a Schrödinger operator, where $\Delta$ is the Laplacian on $\mathbb{R}^{n}$, while nonnegative potential $V$ belongs to the reverse Hölder class. Let also $\Omega \in L_{q}\left(S^{n-1}\right)$ be a homogeneous function of degree zero with $q>1$ and have a mean value zero on $S^{n-1}$. In this paper, we study the boundedness of the Marcinkiewicz operators $\mu_{j, \Omega}^{L}$ and their commutators $\mu_{j, \Omega, b}^{L}$ with rough kernels associated with Schrödinger operator on generalized weighted Morrey spaces $M_{p, \varphi}(w)$. We find the sufficient conditions on the pair $\left(\varphi_{1}, \varphi_{2}\right)$ with $q^{\prime}<p<\infty$ and $w \in A_{p / q^{\prime}}$ or $1<p<q$ and $w^{1-p^{\prime}} \in A_{p^{\prime}} / q^{\prime}$ which ensures the boundedness of the operators $\mu_{j, \Omega}^{L}$ from one generalized weighted Morrey space $M_{p, \varphi_{1}}(w)$ to another $M_{p, \varphi_{2}}(w)$ for $1<p<\infty$. We find the sufficient conditions on the pair $\left(\varphi_{1}, \varphi_{2}\right)$ with $b \in B M O\left(\mathbb{R}^{n}\right)$ and $q^{\prime}<p<\infty, w \in A_{p / q^{\prime}}$ or $1<p<q, w^{1-p^{\prime}} \in A_{p^{\prime}} / q^{\prime}$ which ensures the boundedness of the operators $\mu_{j, \Omega, b}^{L}, j=1, \ldots, n$ from $M_{p, \varphi_{1}}(w)$ to $M_{p, \varphi_{2}}(w)$ for $1<p<\infty$. In all cases the conditions for the boundedness of the operators $\mu_{j, \Omega}^{L}, \mu_{j, \Omega, b}^{L}, j=1, \ldots, n$ are given in terms of Zygmund-type integral inequalities on $\left(\varphi_{1}, \varphi_{2}\right)$ and $w$, which do not assume any assumption on monotonicity of $\varphi_{1}(x, r), \varphi_{2}(x, r)$ in $r$.

Mathematics subject classification (2010): 42B20, 42B35.

Keywords and phrases: Marcinkiewicz operator, rough kernel, Schrödinger operator, generalized weighted Morrey spaces, commutator, $A_{p}$ weights.

\section{REFERENCES}

[1] A. Akbulut, V. S. Guliyev and R. Mustafayev, On the boundedness of the maximal operator and singular integral operators in generalized Morrey spaces, Math. Bohem. 137 (1) (2012), 27-43.

[2] A. AkBulut, O. KUZU, Marcinkiewicz integrals with rough kernel associated with Schrödinger operator on vanishing generalized Morrey spaces, Azerb. J. Math. 4 (1) (2014), 40-54.

[3] J. Alvarez, R. J. Bagby, D. S. Kurtz And C. PÉrez, Weighted estimates for commutators of linear operators, Studia Math. 104 (1993), 195-209.

[4] A. Benedek, A. P. Calderon, R. Panzone, Convolution operators on Banach value functions, Proc. Natl. Acad. Sci. USA 48 (1962), 256-265.

[5] A. P. CALDERON, Commutators of singular integral operators, Proc. Natl. Acad. Sci. USA 53 (1965), 1092-1099.

[6] A. P. CALDERon, Cauchy integrals on Lipschitz curves and related operators, Proc. Natl. Acad. Sci. USA 74 (4) (1977), 1324-1327.

[7] F. ChiarenZa, M. Frasca, Morrey spaces and Hardy-Littlewood maximal function, Rend Mat. 7 (1987), 273-279.

[8] F. Chiarenza, M. Frasca, P. Longo, $W^{2, p}$-solvability of Dirichlet problem for nondivergence elliptic equations with VMO coefficients, Trans. Amer. Math. Soc. 336 (1993), 841-853.

[9] R. CoIfman, R. RochberG, G. Weiss, Factorization theorems for Hardy spaces in several variables, Ann. of Math. 103 (2) (1976), 611-635.

[10] Y. CHEN, Regularity of solutions to elliptic equations with VMO coefficients, Acta Math. Sin. (Engl. Ser.) 20 (2004), 1103-1118. 
[11] Y. Ding, On Marcinkiewicz integral, in: Proc. of the Conference "Singular Integrals and Related Topics, III," Osaka, Japan, 2001, pp. 28-38.

[12] Y. Ding, D. FAn, Y. PAN, Weighted boundedness for a class of rough Marcinkiewicz integrals, Indiana Univ. Math. J. 48 (1999), 1037-1055.

[13] Y. Ding, D. FAn, Y. PAN, $L_{p}$-boundedness of Marcinkiewicz integrals with Hardy space function kernels, Acta Math. Sinica, English Series, 16 (2000), 593-600.

[14] Y. Ding, S. LU, K. YABUtA, On commutators of Marcinkiewicz integrals with rough kernel, J. Math. Anal. Appl, 275 (2002), 60-68.

[15] J. DuOANDikoETXEA, Weighted norm inequalities for homogeneous singular integrals, Trans. Amer. Math. Soc. 336 (1993), 869-880.

[16] J. DZIUbańs Ki, J. Zienkiewicz, Hardy space $H^{1}$ associated to Schrödinger operator with potential satisfying reverse Hölder inequality, Rev. Mat. Iber. 15 (1999), 279-296.

[17] G. Di FAZIO, M. A. RAGUS A, Interior estimates in Morrey spaces for strong solutions to nondivergence form equations with discontinuous coefficients, J. Funct. Anal. 112 (1993), 241-256.

[18] D. FAN, S. LU AND D. YANG, Boundedness of operators in Morrey spaces on homogeneous spaces and its applications, Acta Math. Sinica (N. S.) 14 (1998), suppl., 625-634.

[19] W. GAO, L. TANG, Boundedness for Marcinkiewicz integrals associated with Schrödinger operators, Proc. Indian Acad. Sci. Math. Sci. 124 (2) (2014), 193-203.

[20] M. GIAQUINTA, Multiple integrals in the calculus of variations and nonlinear elliptic systems, Princeton Univ. Press, Princeton, NJ, 1983.

[21] V. S. GULIYEV, Integral operators on function spaces on the homogeneous groups and on domains in $\mathbb{R}^{n}$, Doctoral dissertation, Moscow, Mat. Inst. Steklov, 1994, 329 pp. (in Russian).

[22] V. S. Guliyev, Boundedness of the maximal, potential and singular operators in the generalized Morrey spaces, J. Inequal. Appl. 2009, Art. ID 503948, 20 pp.

[23] V. S. GuliYev, Generalized weighted Morrey spaces and higher order commutators of sublinear operators, Eurasian Math. J. 3 (3) (2012), 33-61.

[24] V. S. Guliyev, S. S. Aliyev, T. Karaman, Boundedness of a class of sublinear operators and their commutators on generalized Morrey spaces, Abstr. Appl. Anal. vol. 2011, Art. ID 356041, 18 pp. doi:10.1155/2011/356041

[25] V. S. GuliYev, S. S. Aliyev, Boundedness of parametric Marcinkiewicz integral operator and their commutators on generalized Morrey spaces, Georgian Math. J. 19 (2012), 195-208.

[26] V. S. GuliYeV, Local generalized Morrey spaces and singular integrals with rough kernel, Azerb. J. Math. 3 (2) (2013), 79-94.

[27] V. S. GULIYEV, Generalized local Morrey spaces and fractional integral operators with rough kernel, J. Math. Sci. (N. Y.) 193 (2) (2013), 211-227.

[28] V. S. Guliyev, T. Karaman, R. Ch. Mustafayev and A. Serbetci, Commutators of sublinear operators generated by Calderón-Zygmund operator on generalized weighted Morrey spaces, Czechoslovak Math. J. 64 (139) (2) (2014), 365-386.

[29] V. S. GUliYeV, M. N. OMAROVA, Higher order commutators of vector-valued intrinsic square functions on vector-valued generalized weighted Morrey spaces, Azerb. J. Math. 4 (2) (2014), 64-85.

[30] L. Hörmander, Translation invariant operators, Acta Math. 104 (1960), 93-139.

[31] S. JANSON, On functions with conditions on the mean oscillation, Ark. Mat. 14 (2) (1976), 189-196.

[32] T. Karaman, V. S. Guliyev, A. Serbetci, Boundedness of sublinear operators generated by Calderón-Zygmund operators on generalized weighted Morrey spaces, Scientic Annals of "Al. I. Cuza" University of Iasi, 60 (1) 2014, 227-244.

[33] Y. Komori AND S. SHIRAI, Weighted Morrey spaces and a singular integral operator, Math. Nachr. 282 (2) (2009), 219-231.

[34] A. Kufner, O. John And S. FuçıK, Function Spaces, Noordhoff International Publishing: Leyden, Publishing House Czechoslovak Academy of Sciences: Prague, 1977.

[35] G. LU, S. LU, D. YANG, Singular integrals and commutators on homogeneous groups, Anal. Math. 28 (2002), 103-134.

[36] T. Mizuhara, Boundedness of some classical operators on generalized Morrey spaces, Harmonic Analysis (S. Igari, Editor), ICM 90 Satellite Proceedings, Springer-Verlag, Tokyo (1991), 183-189.

[37] C. B. Morrey, On the solutions of quasi-linear elliptic partial differential equations, Trans. Amer. Math. Soc. 43 (1938), 126-166. 
[38] B. Muckenhoupt, Weighted norm inequalities for the Hardy maximal function, Trans. Amer. Math. Soc. 165 (1972), 207-226.

[39] Z. SHEN, $L^{p}$ estimates for Schrödinger operators with certain potentials, Ann. Inst. Fourier (Grenoble) 45 (1995), 513-546.

[40] F. SORIA, G. WeISS, A remark on singular integrals and power weights, Indiana Univ. Math. J. 43 (1994), 187-204.

[41] E. M. Stein, On the functions of Littlewood-Paley, Lusin, and Marcinkiewicz, Trans. Amer. Math. Soc. 88 (1958), 430-466.

[42] T. WALSH, On the function of Marcinkiewicz, Studia Math. 44 (1972), 203-217.

[43] H. Xu, J. C. Chen And Y. M. Ying, A note on Marcinkiewicz integrals with $H_{1}$ kernels, Acta. Math. Scientia, Ser. B., 23 (1) (2003) 133-138. 